DOI: 10.1002/adma.((please add manuscript number))

\title{
In-Situ Formation of Crystallographically-Oriented Semiconductor Nanowire Arrays via Selective Vaporization for Optoelectronic Applications
}

Xing Huang*, Yongqiang Yu, Travis Jones, Hua Fan, Lei Wang, Jing Xia, Zhu-Jun Wang, LiDong Shao, Xiang-Min Meng*, and Marc-Georg Willinger*

Dr. X. Huang, H. Fan, L. Wang, J. Xia, Prof. X. M. Meng

Technical Institute of Physics and Chemistry, Chinese Academy of Sciences, 100190, Beijing, P. R. China.

Email: xinghuang0214@mail.ipc.ac.cn; xmmeng@mail.ipc.ac.cn

Dr. X. Huang, Dr. T. Jones, Dr. M. G. Willinger

Fritz Haber Institute of Max Planck Society, Faradayweg 4-6, 14195, Berlin, Germany

Email: willinger@fhi-berlin.mpg.de

Dr. Y. Q. Yu

School of Electronic Science and Applied Physics, Hefei University of Technology, Hefei, 230009, P. R. China

Prof. L. D. Shao

Shanghai Key Laboratory of Materials Protection and Advanced Materials in Electric Power, Shanghai University of Electric Power, P. R. China.

Keywords: in-situ observation; selective vaporization; semiconductor nanowire arrays; cation exchange; photodetector

One dimensional (1D) semiconductor nanowires have drawn considerable interest for the past two decades due to their unique geometry and their novel physical and chemical properties ${ }^{[1,2]}$. A large number of experimental and theoretical studies have demonstrated their potential use as functional components in electronics ${ }^{[3-5]}$, piezoelectrics ${ }^{[6]}$, optoelectronics ${ }^{[7,8]}$, photonics ${ }^{[9}$, ${ }^{10]}$, and mechanics ${ }^{[11,12]}$. As the performances of nanowire-based devices are strongly dependent upon the diameter, length, crystallinity, chemical composition, and structure of nanowires $^{[13-17]}$, precise control over these properties has become the key issue for the ultimate use of the nanowires for various applications ${ }^{[18]}$.

With significant advances in the fabrication techniques over the last number of decades, a variety of routes have been developed for nanowire synthesis, including vapor-liquid--solid 
Submitted to (VLS) growth ${ }^{[19,20]}$, template-based fabrication ${ }^{[21]}$, molecular beam epitaxy $(\mathrm{MBE})^{[22]}$, metal organic vapor phase epitaxy (MOVPE) $)^{[23]}$, oriented-attachment method ${ }^{[24]}$, and e-beam lithography ${ }^{[25]}$. While offering specific advantages, each method has its inevitable weakness and limited applicability. For instance, VLS growth is useful for generating various 1D semiconductor nanowires with controlled size, orientation and composition ${ }^{[26-28]}$, but it needs the assistance of a foreign metal which may introduce contamination in the resultant nanowires $^{[29]}$. Fabrication by MBE, shows merits in regulating composition and structure of uniform nanowires, and yields nanowires of good uniformity with low defect concentration. However, drawbacks such as high cost, low yield, and the requirement of sophisticated procedures and critical conditions make it unsuitable for large-scale application. With the increasing importance of 1D semiconductors in a wide range of areas, development of new and more effective synthetic methods for industrial-scale production of high-quality 1D semiconductor nanowire arrays with controlled orientation and crystal structure are in high demand.

In this work, we introduce an extremely simple approach for large-quantity synthesis of crystallographically-orientated and highly-ordered single crystalline 1D II-VI semiconductor nanowire arrays. It is a top-down, single-step method that only involves heating of wurtzite (WZ) structured semiconductors in a $\mathrm{H}_{2} / \mathrm{Ar}$ atmosphere. In-situ experiments performed in the chamber of an environmental scanning electron microscope (ESEM) clearly demonstrate that the formation of the nanowire arrays proceeds via a selective vaporization process of the bulk crystals. We observe that the vaporization initially leads to a surface roughing and the generation of nanoholes. Selective vaporization leads to carving of nanoholes from both sides of the crystal towards the center until finally, arrays of nanowires remain.

The crystal structure and crystallographic orientation of the resultant nanowires were examined thoroughly by transmission electron microscopy (TEM). It is found that all of the synthesized nanowires are single crystalline and orientated along the [0001] direction of the 


\section{Submitted to

WZ structure. The single crystalline nanowires are free of structural defects, such as dislocation and staking faults or polymorphism, which are commonly present in II-VI group semiconductor nanowires prepared by other methods. Based on the real-time observations, we propose that the selective vaporization behavior of $\mathrm{WZ}$ semiconductors is related to the low symmetry structure of the WZ crystals. A large difference in surface energy among diverse planes could play a role in the selective vaporization process and is confirmed by density functional theory (DFT) calculations. A strong structural sensitivity of this process is confirmed by the fact that the same method applied to zinc-blende (ZB) structured crystals does not lead to the formation of nanowires. On the other hand, co-evaporation of both WZ and ZB structured crystals, for example ZB ZnS and WZ CdS, yields WZ structured ZnS (or $\mathrm{ZnCdS}$ ) nanowire arrays through a cation exchange reaction. In this process, the CdS crystals serve as the WZ structural template for the formation of the nanowire array architecture in which the $\mathrm{Cd}^{2+}$ is gradually replaced by $\mathrm{Zn}^{2+}$ during the selective vaporization of the $\mathrm{CdS}$ crystals. In order to demonstrate the high-quality of the nanowire arrays, we fabricate a photodetector using the as-prepared CdS nanowire arrays. The device exhibits superior optoelectronic performances, including large ON/OFF ratio, fast photoresponse speed, high detectivity, and a large linear dynamic range. We believe that our method represents a transformative new fabrication platform for large-scale fabrication of orientated single crystalline semiconductor nanowire arrays with novel functionalities.

Figure 1a shows a scanning electron microscopy (SEM) image of a CdS bulk crystal used in the experiment. The polished top surface of the crystal appears flat and smooth (Supporting Information, Figure S1). According to the XRD measurement, the sample is a single crystal oriented along the [0001] direction of the WZ structure (Figure 1b). The CdS crystal was placed at the heating zone of a tube furnace and subsequently heated to $750{ }^{\circ} \mathrm{C}$ in a gas mixture of $\mathrm{Ar}$ and $\mathrm{H}_{2}$, as schematically illustrated in Figure S2. The sample was held at $750{ }^{\circ} \mathrm{C}$ for $30 \mathrm{~min}$ and then allowed to cool to room temperature. After the thermal treatment, 


\section{Submitted to

a significant change of the CdS was observed, as seen in the SEM image shown in Figure 1d.

The top surface of the crystal got rougher in comparison to the initial state. Close examination reveals that the roughness of the crystal originates from the formation of nanowires on its surface (Supporting Information, Figure S3a,b). The nanowires show a tapered tip (Figure 1e,f) and a length of about 40 micrometers (Figure S3c). Their base diameter measures about $200 \mathrm{~nm}$. The nanowires are well-aligned and separated from one another (Figure 1g). Due to the evaporation process, the height of the crystal shrank by about 20\% (Figure S4a,b).

TEM was carried out to examine the chemical composition and crystal structure of the asobtained nanowire arrays. An annual dark field scanning TEM (ADF-STEM) image and corresponding EDX elemental mapping of a nanowire are shown in Figure $1 \mathrm{~h}-\mathrm{j}$. It is found that the nanowire consists of $\mathrm{Cd}$ and $\mathrm{S}$ that are evenly distributed over the nanowire. Figure $1 \mathrm{k}$ shows a representative TEM image of CdS nanowires. The nanowires are aligned along their length direction, consistent with our SEM observation. The high-resolution TEM (HRTEM) image of a nanowire is shown in Figure1l. The lattice fringes of the nanowire with $d$-spacings of $0.67 \mathrm{~nm}$ and $0.35 \mathrm{~nm}$ are well-resolved and can be indexed to the (0001) and (01-10) planes of WZ CdS, respectively. The inset shows the corresponding selected-area electron diffraction (SAED) pattern of the nanowire. The diffraction spots appear sharp and strong, thus confirming the high crystallinity of the nanowires. It is worth to mention that the structural defects, such as dislocations or staking faults which are often presented in the polytypic II-VI and III-V semiconductor nanostructures ${ }^{[30-34]}$, are not observed in the current nanowires. Figure $1 \mathrm{~m}$ shows the corresponding atomic model of $\mathrm{CdS}$, viewed in the same orientation as in the HRTEM image. The long axis of the nanowires coincides with the [0001] direction of the WZ structure, and thus, with the original surface normal of the bulk crystal. As a key characteristic of the obtained CdS nanowires, they are aligned, not only in the length direction, but also in the crystallographic orientation. All preceding SEM and TEM characterizations, such as the observed reduction of the thickness of the crystal, the good 
Submitted to

\section{ADVANCED}

alignment of the formed nanowire arrays, and common crystallographic orientation of nanowires, signify that the nanowire array formation might be a result of the direct vaporization of the CdS crystal. However, we cannot completely rule out the possibility that the nanowires could be generated by outward growth via thermal deposition, with the gaseous source originated from the CdS crystal upon heating.

In order to validate the formation mechanism of the nanowire arrays, we performed an in-situ heating experiment in the chamber of an environmental SEM (ESEM), which allows us to directly monitor the growth dynamics of the nanowire arrays. Detailed experimental procedures are described in the experimental section in Supporting Information. A sequence of SEM images obtained during in-situ observation of a $\mathrm{CdS}$ particle under $700^{\circ} \mathrm{C}$ in $\mathrm{H}_{2} / \mathrm{Ar}$ with a pressure of $60 \mathrm{~Pa}$ is shown in Figure 2. It clearly reveals the morphological evolution of the CdS from the particle shape to the nanowire array architecture (Movie M1). At the initial stage, the starting CdS particle shows relatively smooth surfaces except for a few small holes on the top surface. Upon heating, sublimation sets in and leads to surface roughing and the appearance of nanochannels on the top surface (probably also on the bottom surface). With time, the nanochannels increase in depth and proceed gradually towards the center. Finally, highly ordered nanowire arrays are obtained. The in-situ observation confirms that the CdS nanowire arrays are generated by a selective vaporization process rather than by thermal deposition. A parallel ex-situ experiment on the CdS particles was also conducted in this work, which shows a similar result, as displayed in Figure S5a-c. We carried out TEM measurement to explore the fine structure of the CdS nanowire arrays formed from the $\mathrm{CdS}$ particles. As shown in Figure S5d, the nanowires in the bundle are arranged along their length direction. The HRTEM image and fast-Fourier transform (FFT) (Figure S5e,f) further reveal that the nanowire is orientated along the [0001] direction of WZ structure.

To summarize, our ESEM and TEM characterization demonstrate that the formation of CdS nanowire arrays is through the selective vaporization with respect to the crystallography of the 


\section{Submitted to

CdS crystal. It is essentially a top-down approach, but far more simple and efficient compared to the traditional top-down methods. The obtained nanowires are in high quality and high phase purity, and no defects such as dislocations and stalking faults, or polymorphism are observed. The crystallographic orientation of the resultant $\mathrm{CdS}$ nanowires is always along the [0001] direction of the WZ structure, independent of the shape and size of the initial bulk CdS crystals. To the authors' knowledge, direct transformation of a bulk crystal to crystallographically-aligned single crystalline nanowire arrays through only one-step heating has not been reported elsewhere.

Moreover, we find that the method developed for the formation of nanowire arrays works not only on the WZ CdS crystals, but also on the WZ CdSe. Figure 3a shows a SEM image of a raw CdSe particle in a WZ structure (Figure S6a). After a heat treatment of 30 min under $650^{\circ} \mathrm{C}$ in $\mathrm{H}_{2} / \mathrm{Ar}$, arrays of CdSe nanowires were formed (Figure $3 \mathrm{~b}-\mathrm{f}$ ). The cross-section view SEM images clearly show that the nanowires in the arrays are well-separated and oriented along their length. In Figure $3 \mathrm{~b}$, it can be seen that the central part of the particle is not yet completely transformed into the nanowire arrays. This observation is an indication that the transformation initiates from two ends of the particles, which is fully in line with our ESEM observation (Figure 2). More SEM images of the resultant CdSe nanowire arrays are shown in Supporting Information, Figure S6b-d. Chemical analysis demonstrates that the nanowire consists of $\mathrm{Cd}$ and $\mathrm{Se}$, both distributed homogeneously over the entire nanowire (Figure $3 \mathrm{~g}-\mathrm{i}$ ). Structural analysis reveals that the as-prepared CdSe nanowires are orientated along the [0001] direction of the WZ structure (Figure 3j, k), consistent with our observations of the CdS nanowires.

Comparison of the the CdS and CdSe crystals we employed indicates there is a feature common to both of them, i.e., they have the WZ structure. All characteristics of the synthesized $\mathrm{CdX}(\mathrm{X}=\mathrm{S}, \mathrm{Se})$ nanowire arrays, such as high wire-axis alignment, single crystalline nature, and common crystallographic orientation ([0001]), suggest that the 
observed selective vaporization phenomenon is related to the crystallography of the WZ structure of the $\mathrm{CdX}(\mathrm{X}=\mathrm{S}, \mathrm{Se})$. Indeed, we find that the utilization of $\mathrm{ZB}$ structured crystals as raw material does not lead to the formation nanowire arrays or similar 1D nanostructures. Figure S7 shows a sequence of SEM images recorded during in-situ observation of the ZB ZnSe crystal upon heating under $850^{\circ} \mathrm{C}$ in a $\mathrm{H}_{2} / \mathrm{Ar}$ atmosphere $(\mathrm{XRD}$ pattern of the initial $\mathrm{ZB}$ ZnSe crystals is shown in Figure S8). It can be seen that the crystal gradually shrinks from $17.5 \mu \mathrm{m}$ to $5.5 \mu \mathrm{m}$ during the observation (Movie M2). In this process, multiple steps appear on the surface of crystal (insets of Figure S7d-f). As opposed to CdS (Figure 2), the ZB structured ZnSe does not show preferred vaporization behavior.

To further support this result, a parallel ex-situ experiment was carried out in a furnace under a relatively higher temperature $\left(900^{\circ} \mathrm{C}\right)$ in a $\mathrm{H}_{2} / \mathrm{Ar}$ atmosphere. SEM images of the ZnSe after thermal heating are shown in Figure S9d-f. Clearly, nanowires like those shown in Figure 1 were not formed in this case; instead, the terraces and steps on the crystal surface become more pronounced and ordered compared to that of the initial ZnSe crystal (Figure S9a-c). This observation is in agreement with the ESEM observation shown in Figure S7d-f. Additionally, we conducted a heating experiment on another ZB structured crystal, i.e., CdTe (Figure S10ac). Similarly to the case of ZB ZnSe, heating the ZB CdTe did not lead to the formation of nanowires (Figure S10d-e). These results strongly suggest that the direct transformation of bulk material into crystallographically-orientated 1D nanowire arrays is related to the characteristics of WZ structured materials.

We now try to address the intrinsic mechanisms underlying this novel phenomenon. As we showed, the formation of $\mathrm{CdS}$ or $\mathrm{CdSe}$ nanowire arrays is structure sensitive and involves anisotropic vaporization. In-situ SEM observations indicate that the (0001) plane of the crystals decompose much faster than other planes, suggesting that the (0001) plane has the highest reactivity. This is in line with our ab initio atomistic thermodynamics calculations that show the (0001) plane in both WZ CdS and CdSe has the highest surface energy, more than 
two times higher than that of low-index (01-10) and (2-1-10) planes under an $\mathrm{H}_{2}$ atmosphere (Supporting Information, Table S1). These energy differences result in Winterbottom shapes $^{[35]}$ that are consistent with the experimentally observed nanowires (Figure S11a,b). Thus, selective vaporization of the WZ crystals is expected on the basis of energetic considerations.

Next, we address the formation process of the nanowire arrays grown from the bulk crystal. It is known that the system generally tends to reduce its total free energy by minimizing the surfaces area of the high energy surfaces during crystal growth ${ }^{[36]}$. From an energetic perspective, therefore, the high surface energy (0001) plane of the WZ crystal is most likely to reconstruct under elevated temperature to form lower surface energy planes. This has been confirmed by the in-situ experiment, which reveals the roughing process of the top surface under heating, suggesting the reconstruction of the basal plane (Figure 2). After reconstruction, the reformed planes are relatively stable against vaporization. In contrast, the areas enclosed with the remaining (0001) and vicinal planes are more active and are expected to have a faster vaporization rate owing to their higher surface energy ${ }^{[37]}$. In-situ observation during the heating of the CdS crystals shows the random carving of nanoholes on the top surface of the crystal. We speculate that residual (0001) and vicinal planes (or defective sites) in the surface are favorable nucleation sites for vaporization, which leads to the generation of the nanoholes. Once those small holes are created, the vaporization will proceed preferably along them, resulting in a gradual increase of their depth (Figure 2 and Figure S12). Over extended time, highly aligned nanowire arrays with a common crystallographic orientation among nanowires form. Since the nanowires grow from the top to the bottom of the crystal and the preformed parts have longer exposure time for vaporization, the nanowires eventually show a tapered geometry along their length.

As for the $\mathrm{ZB}$ structured $\mathrm{ZnSe}$ and $\mathrm{CdTe}$, the reason for their failure to form nanowires lies in its high symmetry structure and nonuniqueness of high surface energy planes. Our DFT 
Submitted to

\section{ADVANCED}

calculations indicate that there are two sets of planes possessing high surface energy in the ZnSe crystal, they are $\{100\}$ and $\{111\}$ planes, respectively (Table S1). Thus, as the crystals tend to reduce the total surface energy of the system by minimizing the planes with high surface energy, upon heating of ZB crystals, vaporization of high surface energy $\{100\}$ and $\{111\}$ planes will be favored. With time, the area of those planes will be greatly reduced upon heating. As a consequence, the $\{110\}$ facets, which have the lowest surface energy in the ZB structure, are expected to survive. Finally, surface-energy optimized structures are derived, which are in agreement with the Winterbottom shape shown in the Figure S11c.

As we demonstrated above, nanowire arrays are unable to be formed through selective vaporization of $\mathrm{ZB}$ semiconductors because of their high symmetry structure and the similar surface energy of their $\{100\}$ and $\{111\}$ planes. In this regard, arrays of $\mathrm{ZnS}$ nanowires are unachievable from direct vaporization of the ZB structured $\mathrm{ZnS}$ crystals. However, we found that through co-evaporation of powder mixtures of ZB ZnS and WZ CdS (Figure S13), ZnS nanowire arrays with the WZ structure, instead of the ZB structure, can be fabricated. Detailed synthetic procedures are available in experimental section in the Supporting Information. After the reaction, the structure and purity of the product was analyzed by XRD (Figure S14a). As indicated, all of the reflections can be readily indexed according to the hexagonal structured $\mathrm{ZnS}$ with lattice constants of $\mathrm{a}=0.38209 \mathrm{~nm}$ and $\mathrm{c}=0.62573 \mathrm{~nm}$ (JCPDS, No.36-1450).

The morphology of the product was studied with SEM. Figure 4a and Figure S14b show representative SEM images of the ZnS product, and enlarged SEM images of an individual $\mathrm{ZnS}$ structures are shown in Figure S14c-f. It clearly reveals that the $\mathrm{ZnS}$ is comprised of a high density of well-aligned nanowires aligned along their long axis, similar to the synthesized CdS and CdSe nanowire arrays shown in Figure 1 and 3 . It is also worth mentioning that the sizes of these $\mathrm{ZnS}$ nanowire arrays are quite similar to the raw $\mathrm{CdS}$ source employed (Figure S13c,d) and much larger than the particle size of the original ZnS powder 
Submitted to

\section{ADVANCED}

(Figure S13a,b). This indeed is an indication that the $\mathrm{ZnS}$ nanowires form by transformation from the CdS powder, which acts as the template. Furthermore, we investigated the atomic structure of the $\mathrm{ZnS}$ nanowires by TEM. Figure $4 \mathrm{~b}$ shows a typical TEM image of the $\mathrm{ZnS}$ nanowires, and the corresponding SAED pattern is shown in Figure 4c. The TEM image shows that the $\mathrm{ZnS}$ nanowire bundle is made up of individual nanowires aligned along their growth direction, in accordance with the SEM observation. The SAED of the bundles exhibits two sets of diffraction spots, and both of them can be indexed to single crystalline $\mathrm{ZnS}$ with respect to [01-10] and [2-1-10] zone axes of the WZ structure. Atomic modes with different zone axes are shown in Figure $4 \mathrm{f}$ and $\mathrm{i}$, respectively. Nevertheless, all of the nanowires in the bundle orientate along the [0001] direction. HRTEM images with [2-1-10] and [01-10] zone axes were taken to further identify the single crystalline feature of the nanowires. We also investigated the chemical composition of the product by STEM-EDX elemental mapping. The nanowires show elements of $\mathrm{Zn}$ and $\mathrm{S}$ with an atomic ratio of $1: 1$, and they are both homogenously dispersed over the entire length of the nanowires. No Cd signal was detected by EDX, indicating the high purity of the $\mathrm{ZnS}$ nanowires.

According to the above results we propose that there are two processes involved in the formation of $\mathrm{ZnS}$ nanowire arrays, i.e., the selective vaporization of $\mathrm{CdS}$ crystals as well as the cation exchange of $\mathrm{Cd}^{2+}$ by $\mathrm{Zn}^{2+}$. It has been demonstrated by J. Park et al. ${ }^{[38]}$, and also our recent work ${ }^{[39]}$, that the substitution reaction of $\mathrm{Cd}^{2+}$ by $\mathrm{Zn}^{2+}$ is kinetically favorable owing to the smaller radius and the lighter mass of the $\mathrm{Zn}^{2+}$ relative to those of $\mathrm{Cd}^{2+}$. Moreover, because of the lower enthalpy for the formation of $\mathrm{ZnS}$ compared to that for $\mathrm{CdS}$, the chemical transition from $\mathrm{CdS}$ to $\mathrm{ZnS}$ is also thermodynamically favorable. Therefore, it is reasonable to believe that the formation of $\mathrm{ZnS}$ nanowire arrays is through the selective vaporization of $\mathrm{CdS}$ crystals (to form the nanowire array architecture) accompanied by spontaneous cation substitution of $\mathrm{Cd}^{2+}$ by $\mathrm{Zn}^{2+}$. 


\section{Submitted to

To validate our hypothesis, we carried out another heating experiment with a shorter reaction time (30 min). SEM images of the obtained product are shown in Figure S15a-c. As seen, the evolution process of the nanowire arrays is identical to the cases of CdS and CdSe previously shown. In particular, the SEM image shown in Figure S15a again demonstrates that the vaporization process starts from two sides of the crystals and proceeds towards the center. We also carried out STEM-EDX measurement on the nanowires. Elemental analysis reveals that the nanowires bundles consist of $\mathrm{Cd}, \mathrm{Zn}$ and $\mathrm{S}$ elements distribute evenly over the nanowires, see Figure S15d-g. We further performed HRTEM characterization on the synthesized nanowires (Figure S15k-m). All of the nanowires orient along the [0001] direction of the WZ structure. In Figure $\mathrm{S} 15 \mathrm{k}$, the nanowire shows the lattice fringes with a $d$-spacing of about $0.66 \mathrm{~nm}$, which is close to the distance of CdS (0001). In comparison, the nanowire in Figure S151 shows that the (0001) lattice planes have a $d$-spacing of $0.645 \mathrm{~nm}$. This value is in between the distance of $\mathrm{ZnS}$ (0001) and of $\mathrm{CdS}$ (0001), indicating that the $\mathrm{Cd}$ has been partially replaced by $\mathrm{Zn}$ in the nanowire. We also found a nanowire (Figure $\mathrm{S} 15 \mathrm{~m}$ ) showing lattice fringes with a $d$-spacing of about $0.63 \mathrm{~nm}$, which corresponds to the (0001) planes of $\mathrm{ZnS}$. The above evidence verifies the validity of our hypothesis that cation exchange of $\mathrm{Cd}^{2+}$ by $\mathrm{Zn}^{2+}$ is responsible for the $\mathrm{ZnS}$ nanowires formation, which occurs spontaneously with the selective vaporization of CdS crystals.

The array configuration of semiconductor nanowires can contribute to enhanced light absorption due to the light trapping effect, making them extremely attractive for realizing high efficiency optoelectronic devices. In this regard, the as-prepared crystallographicallyorientated CdS nanowire arrays with high density and large aspect ratio were utilized in the fabrication of photodetectors, as illustrated schematically in Figure 5a. The top Schottky contact electrode is a bilayer graphene film, which can facilitate the separation and transport of photogenerated carriers. In particular, the excellent light transparency characteristic of graphene is beneficial for improving the efficiency of light utilization compared to traditional 
metal electrodes. Figure $5 \mathrm{~b}$ shows the spectral response of the device as a function of wavelength. One can see that the maximum spectral response was observed at $\sim 480 \mathrm{~nm}$, close to the bandgap of CdS $(\sim 2.42 \mathrm{eV})$. Figure6c plots the current-voltage $(I-V)$ curves of the device under dark and light illumination in a voltage range of $-5-+5 \mathrm{~V}$. It presents a non-ideal photodiode-like behavior. It is particularly worth noting that the dark current is extremely low and is smaller than that of commercial Si photodetectors and many previously reported 1Dstructure photodetectors ${ }^{[40,41]}$. We believe this behavior is associated with the intrinsic characteristic of the prepared CdS nanowire arrays. As demonstrated by TEM characterization, the nanowire arrays crystallize in high quality with low defect numbers, which ensures the formation of the intrinsic semiconductor instead of the n-type semiconductor. Additionally, the device shows an excellent photoresponse behavior where a rapid increase in photocurrent is observed near the bias of $\sim-0.6 \mathrm{~V}$, as shown in inset of Figure $5 \mathrm{c}$. This is similar to that of the avalanche diode ${ }^{[42]}$, implying a potential use of the device as an avalanche diode with a small breakdown voltage (threshold voltage). A time-dependent photoresponse experiment at fixed bias voltage is also carried out, as shown in Figure 5d. The device is able to give a stable and repeatable current $\mathrm{ON} / \mathrm{OFF}$ ratio $\left(\mathrm{I}_{\mathrm{ON}} / \mathrm{I}_{\mathrm{OFF}}\right)$ of $10^{7}$ and $10^{5}$ at biases of $-5 \mathrm{~V}$ and $0 \mathrm{~V}$, respectively. Particularly, the device can work with high efficiency without applying the bias voltage, signifying that the device can work as a high performance self-powered photodetector. Moreover, the device also shows high sensitivity to the pulsed light with varied frequency from $10 \mathrm{~Hz}$ to $15 \mathrm{kHz}$, as exhibited in Figure S16. The rise time and decay time are determined to be 0.2 and $0.3 \mathrm{~ms}$ at a frequency of $1 \mathrm{kHz}$ (Figure 5e). These characteristics further strengthen the potential of practical application for light wave communication and optical switches.

The specific detectivity $\left(D^{*}\right)$ is a typical figure of merit used to compare the performance of different photodetectors, which is determined by the following formula: $D^{*}=\frac{R}{\left(2 q J_{d}\right)^{1 / 2}}$, where $\mathrm{R}$ is the responsivity, $\mathrm{q}$ is the value of electron charge, $J_{\mathrm{d}}$ is the dark current density. $D^{*}$ is 


\section{ADNANED
MAATRRALIS}

calculated to be $1.86 \times 10^{13}$ Jones (Jones $=\mathrm{cm} \mathrm{Hz}^{1 / 2} \mathrm{~W}^{-1}$ ). This value is more than one order of magnitude larger than those obtained from 1D and 2D materials based photodetectors $\left(10^{7}\right.$ $10^{12}$ Jones $)^{[43,44]}$. Since the specific detectivity can give a fair comparison of different photodetectors by normalizing the device area ${ }^{[45]}$, the higher value of the device in the current work indicates great potential of the $\mathrm{CdS}$ nanowire arrays for high performance optoelectronic applications. Another figure of merit for photodetectors is the linear dynamic range (LDR, typically quoted in $\mathrm{dB}$ ), which characterizes the light intensity range where the responsivity remains constant. The device yields a LDR of $140 \mathrm{~dB}$ at a bias of $-5 \mathrm{~V}$ based on the following equation: $L D R=20 \log \left(I_{p} / I_{d}\right)$. This result is higher than 2D Transition Metal Dichalcogenide based photodetectors $(40-80 \mathrm{~dB})^{[46]}$ as well as the commercial Si photodetectors $(120 \mathrm{~dB})$. Noise equivalent power (NEP), representing the minimum optical input power that a device can distinguish from the noise, is also an important parameter for evaluating photodetectors, which can be expressed as NEP $=i_{n} / \mathrm{R}$. To determine the NEP value, the noise current $\left(i_{n}\right)$ of the device was measured at varied frequency under the bias of $-5 \mathrm{~V}$, as shown in Figure $5 f$. The calculated NEP value is as low as $4.7 \times 10^{-11} \mathrm{~W}$ at $-5 \mathrm{~V}(800 \mathrm{~Hz})$, suggesting that the device is capable of weak light detection. The main characteristic parameters of the Graphene/CdS nanowire arrays based photodetector from the current study, along with other 1D and 2D semiconductor based photodetectors from previous reports, are summarized in Table 1. One can clearly see that the Graphene/CdS nanowire arrays exhibit superior performance, such as higher detectivity, larger LDR, and lower dark current, surpassing most other 1D and 2D semiconductors for high-performance optoelectronic applications.

In conclusion, we demonstrated a facile and efficient method for large-scale fabrication of crystallographically-oriented and highly-aligned II-VI semiconductor nanowire arrays in the WZ structure. In-situ experiments performed in the chamber of an ESEM revealed that the nanowire arrays were formed through a selective vaporization process. Furthermore, we found 


\section{ADVANCEP}

Submited to MATERIALS

that the selective vaporization behavior has a structural dependency, and only occurs in WZ structured crystals, such as CdS and CdSe. The crystallographic orientation of the resultant $\mathrm{CdS}$ and CdSe nanowires is always along the [0001] direction of the WZ structure as demonstrated by TEM characterization and is independent of the shape and size of the initial crystals. In addition, through a cation exchange of $\mathrm{Cd}^{2+}$ by $\mathrm{Zn}^{2+}$, WZ $\mathrm{ZnS}$ nanowire arrays can also be grown through co-evaporation of powder mixture of $\mathrm{WZ} \mathrm{CdS}$ and $\mathrm{ZB} \mathrm{ZnS}$. In this process, the CdS crystal serves as a WZ structural template for the formation of nanowire arrays. Remarkably, the optoelectronic device fabricated with the nanowire arrays exhibits excellent operating performances, such as a large on/off ratio of $10^{7}$, high detectivity of 1.86 $\times 10^{13}$ Jones, a high linear dynamic range of $140 \mathrm{~dB}$, low dark current of $20 \mathrm{pA}$, and fast photoresponse speed (rise time and decay time are 0.2 and $0.3 \mathrm{~ms}$, respectively). Our research opens up a new pathway to fabricate single crystalline semiconductor nanowire arrays, featured with high chemical and phase purity, high crystallinity and consistent orientation, for various electronic, optoelectronic and photovoltaic applications.

\section{Supporting Information}

Supporting Information is available from the Wiley Online Library or from the author.

\section{Acknowledgements}

We acknowledge the financial support from "Strategic Priority Research Program" of Chinese Academy of Sciences (Grant No.XDA09040203) and 973 Project (2012CB932401). Dr. X. Huang would like to thank the financial support from doctoral training program between Chinese Academy of Sciences and Max Planck Society during the period of 2011-2012.

Received: ((will be filled in by the editorial staff))

Revised: ((will be filled in by the editorial staff)) Published online: ((will be filled in by the editorial staff))

[1] N. P. Dasgupta, J. W. Sun, C. Liu, S. Brittman, S. C. Andrews, J. Lim, H. W. Gao, R. X. Yan, P. D. Yang, Adv. Mater., 2014, 26, 2137-2184.

[2] Z. Liu, J. Xu, D. Chen, G. Z. Shen, Chem. Soc. Rev., 2015, 44, 161-192.

[3] J. Xiang, W. Lu, Y. J. Hu, Y. Wu, H. Yan, C. M. Lieber, Nature, 2006, 441, 489-493. 
[4] F. Xu, W. Lu, Y. Zhu, ACS Nano, 2011, 5, 672-678.

Submitted to

[5] X. M. Zou, J. L. Wang, X. Q. Liu, C. L. Wang, Y. Jiang, Y. Wang, X. H. Xiao, J. C. Ho, J. C. Li, C. Z. Jiang, Y. Fang, W. Liu, L. Liao, Nano Letters, 2013, 13, 3287-3292.

[6] Z. L. Wang, J. H. Song, Science, 2006, 312, 242-246.

[7] X. Huang, Y. Q. Yu, J. Xia, H. Fan, L. Wang, M. G. Willinger, X. P. Yang, Y. Jiang, T. R. Zhang, X. M. Meng, Nanoscale, 2015, 7, 5311-5319.

[8] W. Tian, T. Y. Zhai, C. Zhang, S. L. Li, X. Wang, F. Liu, D. Q. Liu, X. K. Cai, K. Tsukagoshi, D. Golberg, Y. Bando, Adv. Mater., 2013, 25, 4625-4630.

[9] T. Kuykendall, P. Ulrich, S. Aloni, P. Yang, Nat. Mater., 2007, 6, 951-956.

[10] C. F. Pan, L. Dong, G. Zhu, S. M. Niu, R. M. Yu, Q. Yang, Y. Liu, Z. L. Wang, Nat. Photonics, 2013, 7, 752-758.

[11] S. Z. Yang, L. F. Wang, X. Z. Tian, Z. Xu, W. L. Wang, X. D. Bai, E. G. Wang, Adv. Mater., 2012, 24, 4676-4682.

[12] G. M. Cheng, C. Y. Miao, Q. Q. Qin, J. Li, F. Xu, H. Haftbaradaran, E. C. Dickey, H. J. Gao, Y. Zhu, Nat. Nanotechnol., 2015, 10, 687-691.

[13] A. Soudi, C. H. Hsu, Y. Gu, Nano Lett., 2012, 12, 5111-5116.

[14] M. L. Ren, W. J. Liu, C. O. Aspetti, L. X. Sun, R. Agarwal, Nat. Commun., 2014, 5, 8.

[15] L. F. Hu, J. Yan, M. Y. Liao, H. J. Xiang, X. G. Gong, L. D. Zhang, X. S. Fang, Adv. Mater., 2012, 24, 2305-2309.

[16] X. Huang, M. Wang, M. G. Willinger, L. D. Shao, D. S. Su, X. M. Meng, ACS Nano, 2012, 6, 7333-7339.

[17] P. Krogstrup, N. L. B. Ziino, W. Chang, S. M. Albrecht, M. H. Madsen, E. Johnson, J. Nygard, C. M. Marcus, T. S. Jespersen, Nat. Mater., 2015, 14, 400-406.

[18] Y. N. Xia, P. D. Yang, Y. G. Sun, Y. Y. Wu, B. Mayers, B. Gates, Y. D. Yin, F. Kim, Y. Q. Yan, Adv. Mater., 2003, 15, 353-389.

[19] R. S. Wagner, W. C. Ellis, Appl. Phys. Lett., 1964, 4, 89-90.

[20] Y. Y. Wu, P. D. Yang, J. Am. Chem. Soc., 2001, 123, 3165-3166.

[21] U. Y. Jeong, Y. N. Xia, Y. D. Yin, Chem. Phys. Lett., 2005, 416, 246-250.

[22] A. Sarwar, S. D. Carnevale, T. F. Kent, M. R. Laskar, B. J. May, R. C. Myers, J. Cryst. Growth, 2015, 428, 59-70.

[23] P. Gibart, Rep. Prog. Phys., 2004, 67, 667-715.

[24] N. Pradhan, H. F. Xu, X. G. Peng, Nano Lett., 2006, 6, 720-724.

[25] R. Juhasz, N. Elfstrom, J. Linnros, Nano Lett., 2005, 5, 275-280.

[26] T. R. Kuykendall, M. V. P. Altoe, D. F. Ogletree, S. Aloni, Nano Lett., 2014, 14, 6767-6773.

[27] M. T. Borgstrom, G. Immink, B. Ketelaars, R. Algra, E. Bakkers, Nat. Nanotechnol., 2007, 2, 541-544.

[28] H. Wang, J. T. Wang, Z. X. Cao, W. J. Zhang, C. S. Lee, S. T. Lee, X. H. Zhang, Nat. Commun., $2015,6,7$.

[29] J. B. Hannon, S. Kodambaka, F. M. Ross, R. M. Tromp, Nature, 2006, 440, 69-71.

[30] H. Heo, K. Kang, D. Lee, L. H. Jin, H. J. Back, I. Hwang, M. Kim, H. S. Lee, B. J. Lee, G. C. Yi, Y. H. Cho, M. H. Jo, Nano Letters, 2012, 12, 855-860.

[31] F. Meng, M. Estruga, A. Forticaux, S. A. Morin, Q. Wu, Z. Hu, S. Jin, Acs Nano, 2013, 7, 11369-11378.

[32] P. Caroff, K. A. Dick, J. Johansson, M. E. Messing, K. Deppert, L. Samuelson, Nature Nanotechnology, 2009, 4, 50-55.

[33] X. Z. Yu, H. L. Wang, J. Lu, J. H. Zhao, J. Misuraca, P. Xiong, S. von Molnar, Nano Letters, 2012, 12, 5436-5442.

[34] F. D. Wang, W. E. Buhro, Nano Letters, 2016, 16, 889-894.

[35] W. L. Winterbottom, Acta Metallurgica, 1967, 15, 303-310.

[36] J. I. Sohn, W. K. Hong, S. Lee, S. Lee, J. Ku, Y. J. Park, J. Hong, S. Hwang, K. H. Park, J. H. Warner, S. Cha, J. M. Kim, Sci Rep, 2014, 4, 7.

[37] D. J. Hellebusch, K. Manthiram, B. J. Beberwyck, A. P. Alivisatos, J. Phys. Chem. Lett., 2015, 6, 605-611.

[38] J. Y. Lee, D. S. Kim, J. H. Park, Chem. Mat., 2007, 19, 4663-4669.

[39] X. Huang, Z. J. Wang, G. Weinberg, X. M. Meng, M. G. Willinger, Adv. Funct. Mater., 2015, 25, 5979-5987. 
Submitted to

[40] C. Yang, C. J. Barrelet, F. Capasso, C. M. Lieber, Nano Lett., 2006, 6, 2929-2934.

[41] J. S. Jie, W. J. Zhang, I. Bello, C. S. Lee, S. T. Lee, Nano Today, 2010, 5, 313-336.

[42] B. Zhao, F. Wang, H. Y. Chen, Y. P. Wang, M. M. Jiang, X. S. Fang, D. X. Zhao, Nano Lett., 2015, 15, 3988-3993.

[43] W. Choi, M. Y. Cho, A. Konar, J. H. Lee, G. B. Cha, S. C. Hong, S. Kim, J. Kim, D. Jena, J. Joo, S. Kim, Adv. Mater., 2012, 24, 5832-5836.

[44] C. Xie, B. Nie, L. H. Zeng, F. X. Liang, M. Z. Wang, L. B. Luo, M. Feng, Y. Q. Yu, C. Y. Wu, Y. C. Wu, S. H. Yu, ACS Nano, 2014, 8, 4015-4022.

[45] F. W. Guo, Z. G. Xiao, J. S. Huang, Adv. Opt. Mater., 2013, 1, 289-294.

[46] P. A. Hu, L. F. Wang, M. Yoon, J. Zhang, W. Feng, X. N. Wang, Z. Z. Wen, J. C. Idrobo, Y. Miyamoto, D. B. Geohegan, K. Xiao, Nano Lett., 2013, 13, 1649-1654.

[47] B. A. Nie, J. G. Hu, L. B. Luo, C. Xie, L. H. Zeng, P. Lv, F. Z. Li, J. S. Jie, M. Feng, C. Y. Wu, Y. Q. Yu, S. H. Yu, Small, 2013, 9, 2872-2879.

[48] L. B. Luo, J. J. Chen, M. Z. Wang, H. Hu, C. Y. Wu, Q. Li, L. Wang, J. A. Huang, F. X. Liang, Adv. Funct. Mater., 2014, 24, 2794-2800.

[49] P. Lv, X. J. Zhang, X. W. Zhang, W. Deng, J. S. Jie, IEEE Electron Device Lett., 2013, 34, 13371339.

[50] L. T. Dou, Y. Yang, J. B. You, Z. R. Hong, W. H. Chang, G. Li, Y. Yang, Nat. Commun., 2014, 5,6 .

[51] O. Lopez-Sanchez, D. Lembke, M. Kayci, A. Radenovic, A. Kis, Nat. Nanotechnol., 2013, 8, 497-501. 


\section{Figures and captions}

\section{ADVANCED \\ Submitted to \\ MATERIALS}
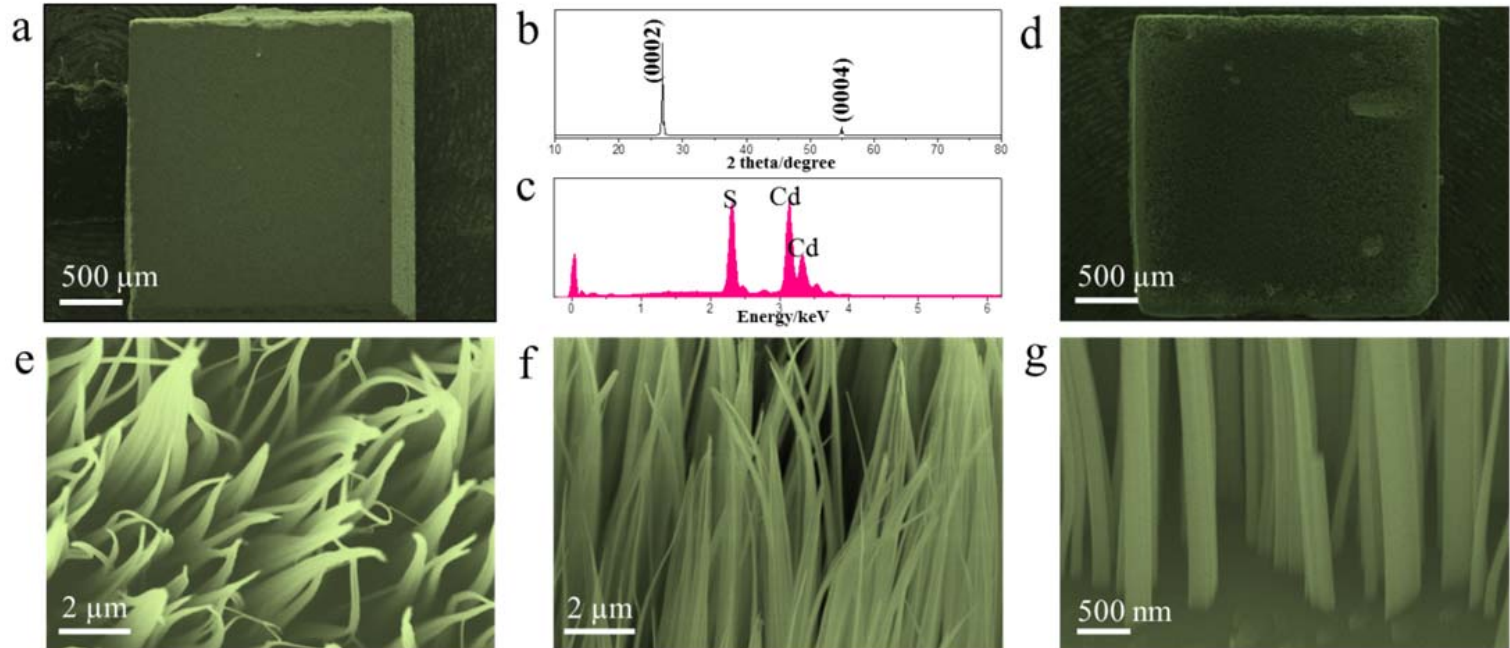

$500 \mu \mathrm{m}$
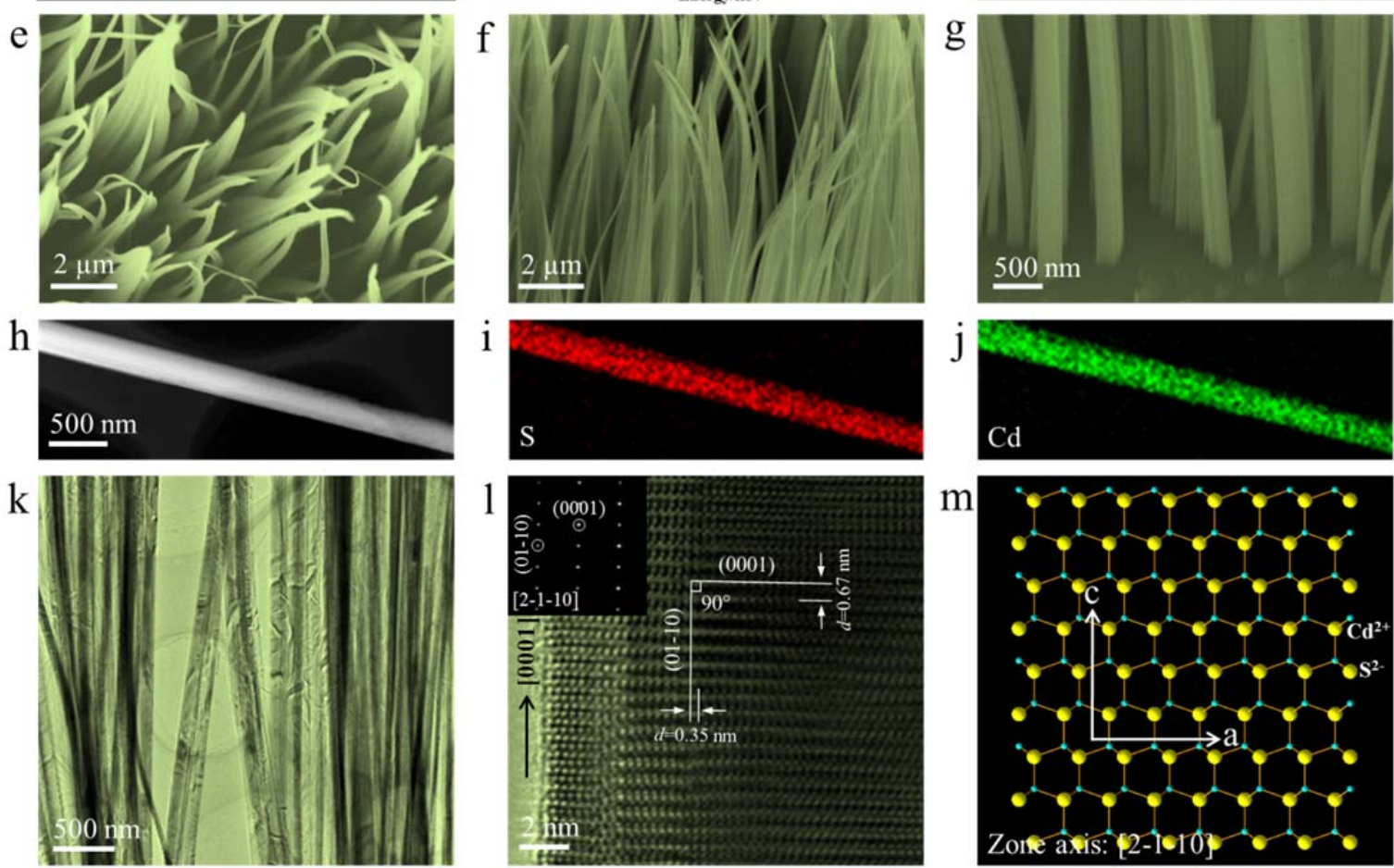

Figure 1. Morphology, structure and composition analyses of CdS nanowire arrays transformed from the CdS single crystal. (a) SEM image of the initial CdS crystal; $(b, c)$ XRD pattern and EDX spectrum of the CdS crystal. Top-view (d,e) and tilted-view (f,g) SEM images of the crystal after heating, showing the morphology of the resulting nanowire arrays; (h) ADF-STEM image and (i,j) corresponding EDX elemental mapping of $\mathrm{S}$ and $\mathrm{Cd}$; $(\mathrm{k}, \mathrm{l})$ TEM image and HRTEM image of the synthesized CdS nanowires, inset of Figure1m shows the SAED pattern of the nanowire; (m) Atomic model of nanowire with a zone axis of [2-1-10], corresponding to the HRTEM image. 


\section{ADVANCED \\ Submitted to}
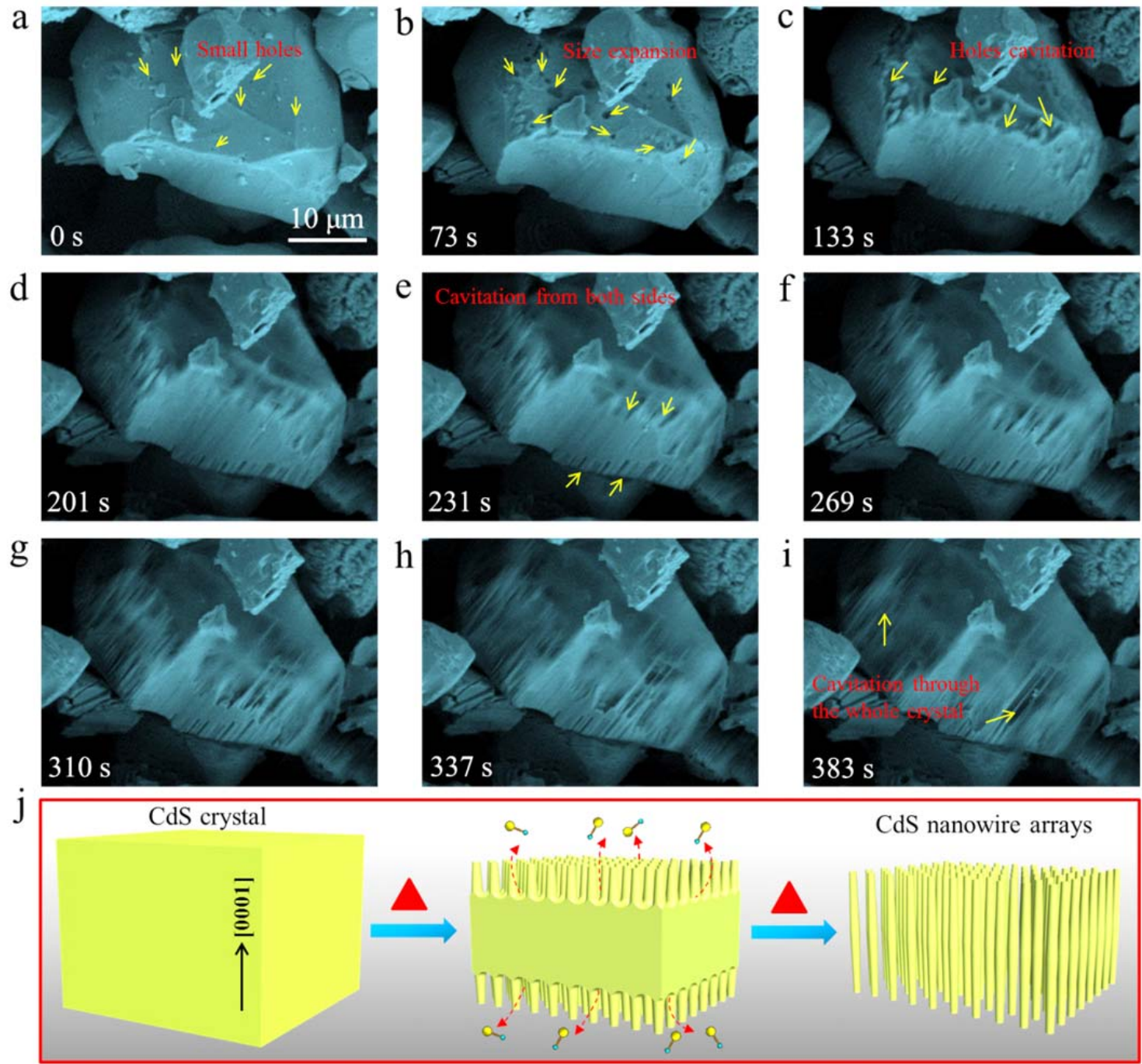

Figure 2. In-situ observation during the formation of CdS nanowire arrays. (a-i) A series of SEM images recorded during in-situ observation of CdS crystals upon heating in an $\mathrm{H}_{2} / \mathrm{Ar}$ atmosphere, showing direct kinetics of formation of highly aligned nanowire arrays; (j) schematic showing the formation process of the nanowire arrays. 


\section{ADVANCED \\ Submitted to \\ MATERIALS}

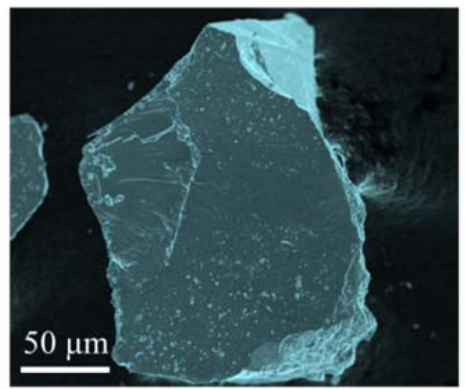

d

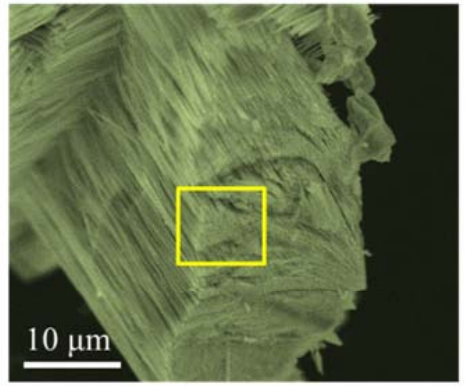

$\mathrm{g}$

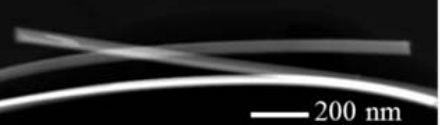

$\mathrm{j}$

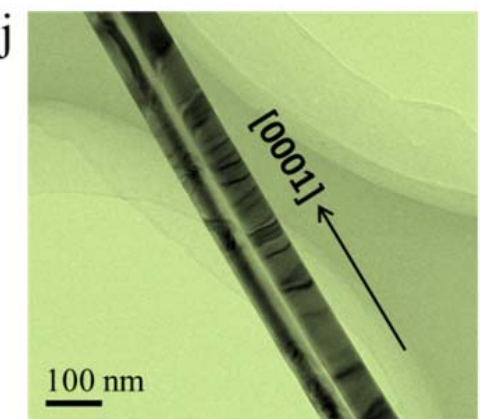

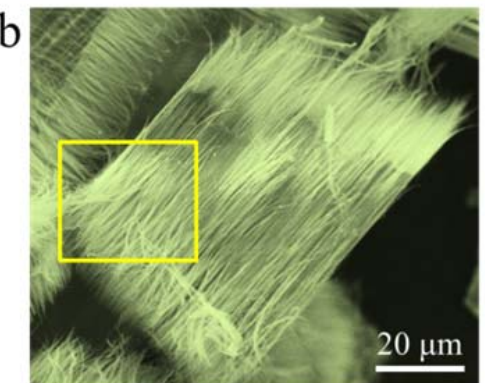
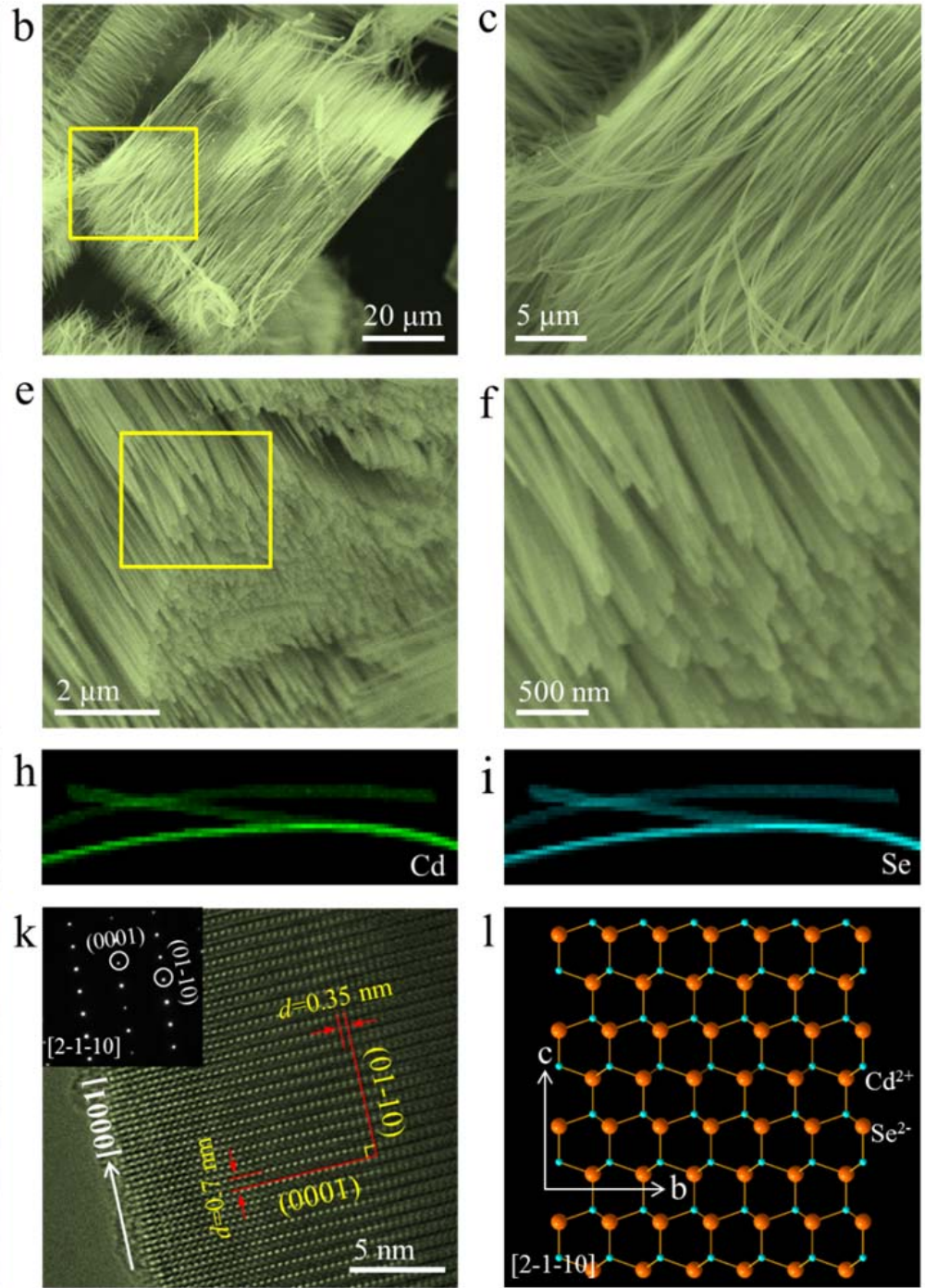
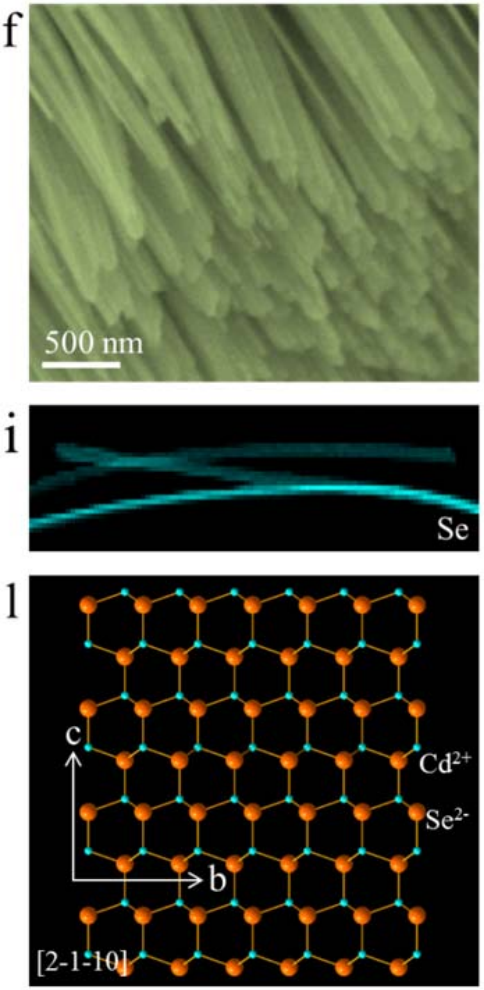

Figure 3. Microscopic analysis of as-prepared CdSe nanowire arrays. (a) SEM image of the initial CdSe crystals; (b-f) SEM images of the CdSe nanowires obtained by heating the raw CdSe crystals; (g-i) ADF-STEM image and corresponding EDX elemental mapping of $\mathrm{Cd}$ and Se; (j,k) TEM image and HRTEM image of the synthesized CdSe nanowires, inset of Figure3k shows the SAED pattern of the nanowire; (1) Atomic mode of the CdSe with a zone-axis of [2-1-10]. 


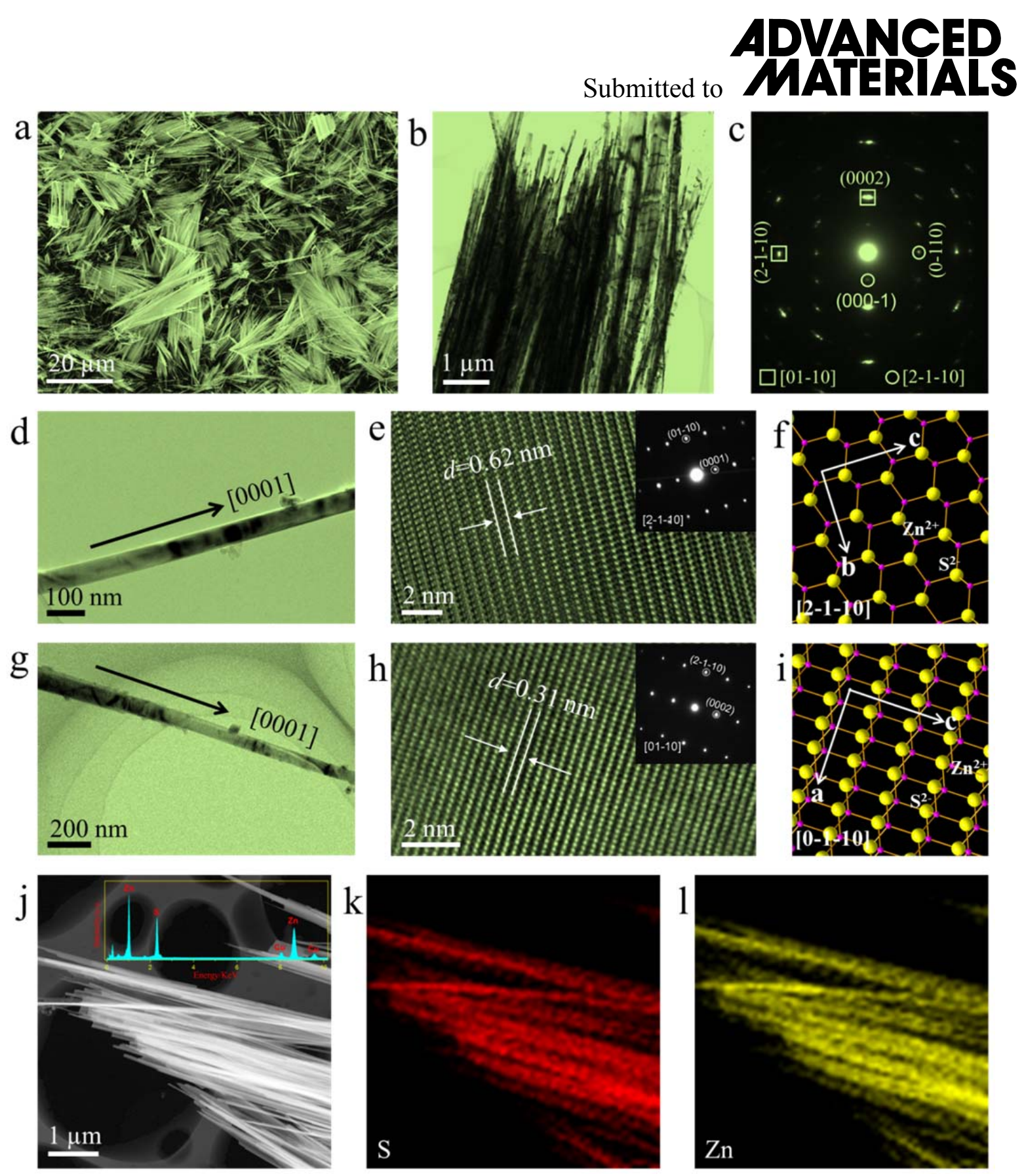

Figure 4. Characterization of the nanowire arrays after co-heating of ZB ZnS and WZ CdS powder, showing the formation of WZ ZnS nanowire arrays. (a) SEM images of the synthesized $\mathrm{ZnS}$ nanowire arrays; (b) TEM images of the ZnS nanowires; (c) SAED pattern of the nanowires bundles; (d,e) TEM image of a ZnS nanowire and corresponding HRTEM image taken from the [2-110] projection, inset of Figure 4e shows the SAED pattern of the nanowire; (g,h) TEM image of a $\mathrm{ZnS}$ nanowire and its HRTEM image taken from the [01-10] projection, inset of Figure 4 h shows the SAED pattern of the nanowire; ( $\mathrm{j}-1)$ ADF-STEM image of a $\mathrm{ZnS}$ nanowires bundle and corresponding EDX elemental mapping of $\mathrm{S}$ and $\mathrm{Zn}$, inset Figure 4j shows the EDX spectrum of the nanowires bundle; (f,i) Atomic models corresponding to the HRTEM images of (e,h) with a zone-axis of [2-1-10] and [01-10], respectively. 


\section{ADNANEDS
MATERRALIS}

a
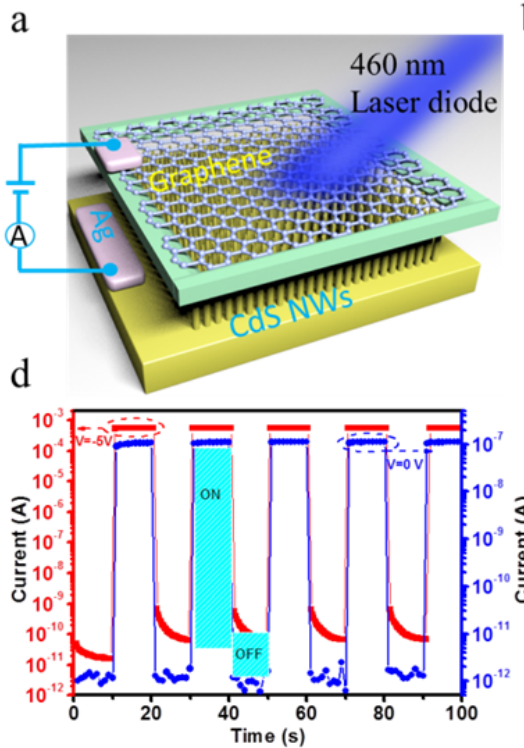

$\mathrm{b}$
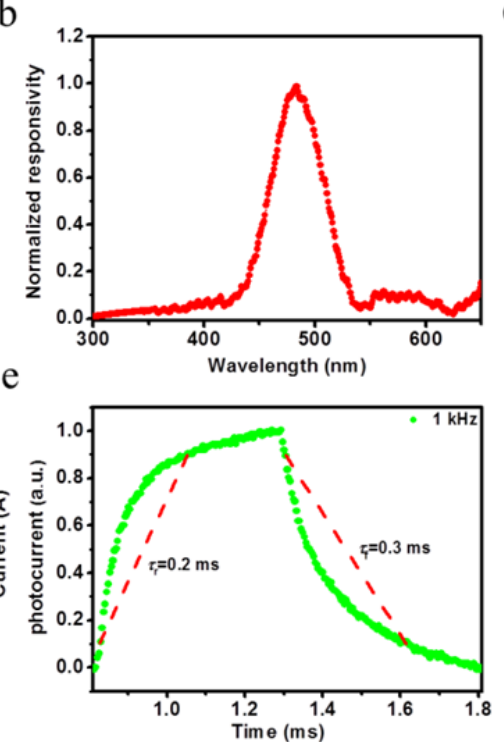

$\mathrm{c}$

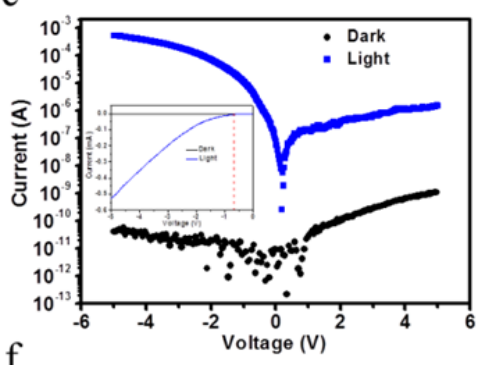

$\mathrm{f}$

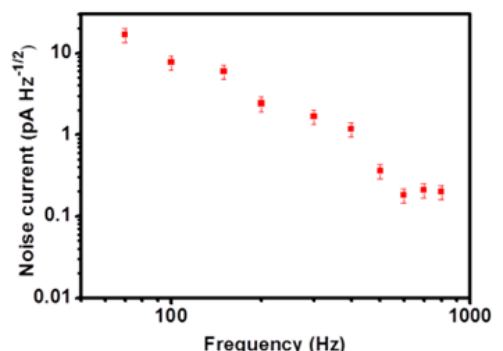

Figure 5. Optoelectronic characterization of the device fabricated with CdS nanowire arrays. (a)

Schematic illustration of the structure of the Graphene/CdS NWs diode photodetector. (b) Spectral response of the device in the range of $300 \mathrm{~nm}-650 \mathrm{~nm}$. (c) $I-V$ curves of the photodetector measured under the dark and light illumination $\left(460 \mathrm{~nm}, 39 \mathrm{mWcm}^{-2}\right)$, respectively. (d) Time response of the photodetector under light illumination at bias voltage of $-5 \mathrm{~V}$ and $0 \mathrm{~V}$, respectively. (e) Normalized photoresponse characteristic of the photodetector at a pulsed frequency of $1 \mathrm{kHz}$. (f) Dark current noise $\left(i_{\mathrm{n}}\right)$ measured at different frequencies at bias of $-5 \mathrm{~V}$. 


\section{Submitted to

Table 1. Comparison of characteristic parameters for the photodetectors from present Graphene/CdS nanowire arrays and those from previous reports.

\begin{tabular}{|c|c|c|c|c|c|c|}
\hline Device structure & $\begin{array}{l}\text { Working } \\
\text { voltage }\end{array}$ & $\begin{array}{l}\text { Responsivity } \\
\left(\mathrm{AW}^{-1}\right)\end{array}$ & $\begin{array}{c}\text { Detectivity } \\
\text { (Jones) }\end{array}$ & $\mathrm{LDR}(\mathrm{dB})$ & $\begin{array}{c}\text { Dark } \\
\text { current }\end{array}$ & Ref. \\
\hline $\begin{array}{c}\text { Graphene/CdS } \\
\text { nanowire arrays }\end{array}$ & $-5 \mathrm{~V}$ & 0.43 & $1.9 \times 10^{13}$ & 140 & $20 \mathrm{pA}$ & $\begin{array}{r}\text { Our } \\
\text { work }\end{array}$ \\
\hline Si NWs/CQD & $0 \mathrm{~V}$ & 0.35 & $3.7 \times 10^{9}$ & $\sim 60$ & $\sim 30 \mathrm{nA}$ & [44] \\
\hline $\begin{array}{l}\text { Graphene/ZnO } \\
\text { nanorod arrays }\end{array}$ & $-5 \mathrm{~V}$ & 113 & - & 60 & $\sim 1 \mu \mathrm{A}$ & [47] \\
\hline $\begin{array}{l}\text { Graphene/GaAs } \\
\text { nanocone arrays }\end{array}$ & $0 \mathrm{~V}$ & $3.7 \times 10^{-3}$ & $1.8 \times 10^{11}$ & 80 & $\begin{array}{c}0.1 \\
\mathrm{nA}\end{array}$ & [48] \\
\hline Graphene/Si & $0 \mathrm{~V}$ & $\sim 0.5$ & $3.9 \times 10^{11}$ & 40 & - & [49] \\
\hline Perovskite & $-1 \mathrm{~V}$ & $\sim 1.0$ & $\sim 10^{12}$ & 100 & $\sim 1 \mathrm{nA}$ & [50] \\
\hline $\begin{array}{l}\text { Single-layer } \\
\mathrm{MoS}_{2}\end{array}$ & $\begin{array}{c}8 \mathrm{~V} \\
\mathrm{Vg}=-70 \mathrm{~V}\end{array}$ & 880 & - & 120 & $\sim 50 \mathrm{pA}$ & [51] \\
\hline
\end{tabular}

